\title{
The possibilities for comprehensive studies involving the Thirty Meter Telescope of the 'zoo' of high energy sources.
}

\author{
Warren Skidmore* \\ Thirty Meter Telescope International Observatory, 100 West Walnut Street, Suite 300, Pasadena, \\ CA 91105, USA \\ E-mail: wasetmt.org
}

\begin{abstract}
The Thirty Meter Telescope (TMT) will provide exciting capabilities for the study of high energy sources of many different types. Future ground based general purpose extremely large telescopes (ELTs) have important roles to play in studies of low mass X-ray binaries (LMXBs) and neutron stars, super novae and gamma-ray bursts (GRBs), super-massive black holes (SMBH) and feedback and other processes in their host galaxies, colliding galaxies and dark matter. From the perspective of TMT, I describe a selection of possible studies that will illustrate the capabilities that the TMT (and the other future ELTs) will provide such as support for ToO observations, time resolved and cadence observations and the importance of coordinated observations with other facilities. I give an overview of the timeline for the beginning of TMT operations and the flexible selection and development process for new instruments.
\end{abstract}

XII Multifrequency Behaviour of High Energy Cosmic Sources Workshop

12-17 June, 2017

Palermo, Italy

\footnotetext{
${ }^{*}$ Reporting aspects of the 2015 TMT Detailed Science Case [1] which was compiled by members of the TMT International Science Teams, Science Advisory Committee and others.
} 


\section{Introduction to TMT}

The Thirty Meter Telescope (TMT) is one of three general purpose extremely large ground based telescopes (ELTs) planned to begin operations in the mid-2020s. The 39m European Extremely Large Telescope (E-ELT) and 24.5/22m Giant Magellan Telescope are being constructed in Chile. The TMT will be constructed in the northern hemisphere, either on Maunakea, Hawaii or at Observatorio del Roque de los Muchachos, La Palma and operations are planned to start in the late 2020s.

The ELTs form part of the suite of facilities that will be available for astronomers in the future, providing a range of key capabilities alongside wide angle survey telescopes, radio, X-ray and $\gamma$ ray telescopes and gravitational wave observatories. Exciting possibilities exist for research that bridges between astrophysics to high-energy and particle physics.

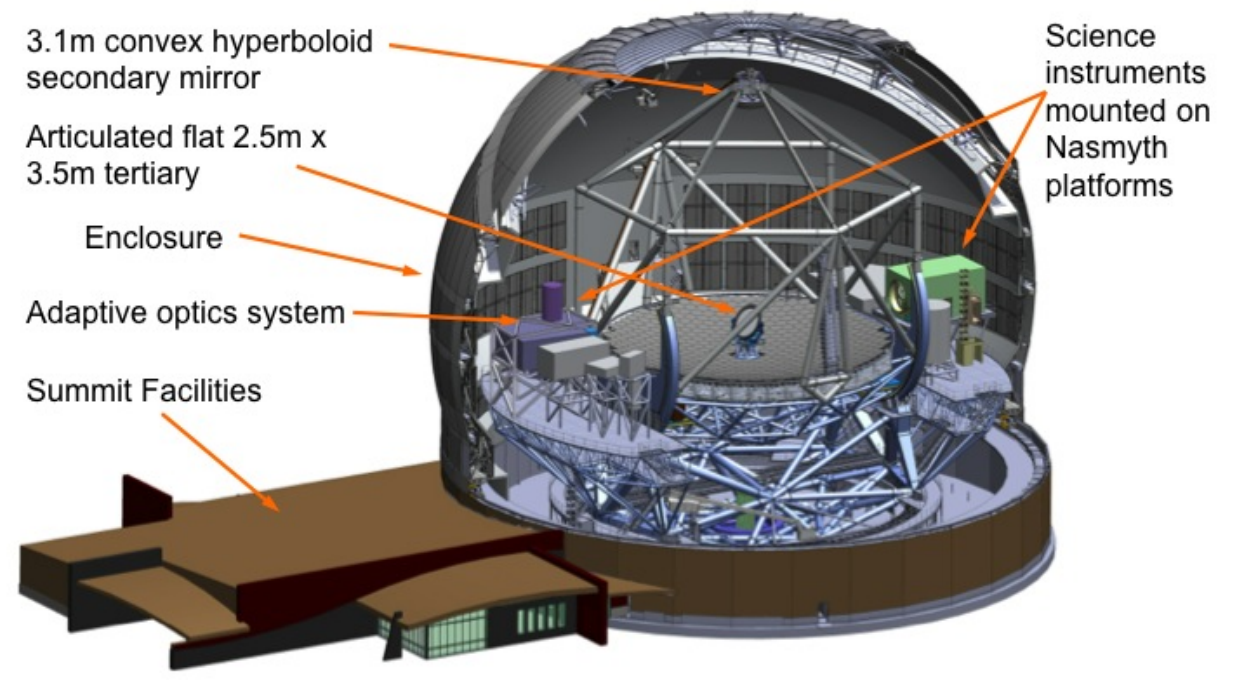

Figure 1: An overview of the design for the Thirty Meter Telescope Observatory. The Ritchey-Chrétien design has an effective f/15 final focal ratio with a 20" field of view, $2.62 \mathrm{~m}$ diameter focal plane with 0.46 milli-arcsends $/ \mu \mathrm{m}$ pixel scale. Instruments have fixed gravity vector by being located on the Nasmyth platforms and any instrument can be selected by rotating the tertiary mirror. The first light adaptive optics system can feed up to 3 instruments and up to about 10 instruments (depending on instrument choices) can be accommodated on the Nasmyth platforms. (Credit: TMT International Observatory)

The TMT Observatory is being designed to support both queue scheduled and classical (i.e. astronomers directly involved) observing modes. A mix of small programs for individual PIs and large cross partner key programs will be carried out. Remote operations and eavesdropping modes will be provided so that principal investigators (PIs) can participate in or monitor the gathering of data by observatory staff without traveling to the observatory. Flexible scheduling will be used to 
optimize science output based on the atmospheric conditions and priorities of observing programs in the queue.

The TMT International Observatory partnership presently consists of India, Canada, Japan, China, the University of California and Caltech. Each partner is responsible for delivering various subsystems within the observatory. At the time of writing, all critical sub systems are either in construction or in the final design phase and development is proceeding in all areas necessary for delivering an operational observatory in the latter part of the 2020s.

\section{Science areas in which TMT is envisioned to make contributions}

\subsection{Baryonic Power Spectrum on the Smallest Scales}

The large increase in sensitivity provided by TMT will allow the measurement of the Lyman- $\alpha$ forest with a much greater density of sight lines. By using faint background galaxies as the continuum sources then about 200x greater spatial density will be achieved compared to that presently achieved with quasars. Significantly improved measurements of the Lyman- $\alpha$ forest will allow much more stringent constraints to be placed on the ratio of cold to hot dark matter and reconcile the ratio with the observed Baryon fraction.

On a more fundamental level, determining the high spatial frequency fluctuations in the baryonic power spectrum, quantifying deviations in the power spectrum from unity on spatial scales much smaller than probed by Planck, will provide important information on the fields that drove inflation.

\subsection{Studying Dark Energy with Time-delay Cosmography}

Dark energy is driving the acceleration of the expansion of the universe. A major problem facing modern cosmology is distinguishing between different possible dark energy models. Different dark energy models have different equations of state but there is always a degeneracy between the equation of state and the cosmic curvature parameter. To break this degeneracy we need to measure both the time delays and the angular separations between multiple images of gravitationally lensed quasars and supernovae using high spatial resolution imaging (see Figure 2), correlating these with redshift. This technique will be more successful when combined with cosmic microwave background measurements. The redshifts to the lensed quasars and super novae and the internal dynamics (and hence the gravitational mass) and redshift of the gravitational lens will be obtained using diffraction limited high spatial resolution spectroscopy with spectral resolution of $\mathrm{R} \sim 5000$.

\subsection{Dark Matter self-interaction cross-section}

Ground based experiments searching for the interaction of dark matter particles with baryonic matter have so far been unsuccessful. Astrophysical studies allow the interactions between dark matter particles themselves to be examined by studying the the structure and dynamics of dark matter halos in clusters of interacting galaxies.

The characteristics of the dark matter halo are determined using gravitationally lensed background tracers and dynamical studies of stellar and gaseous sources, see Figure 3. Determining a 


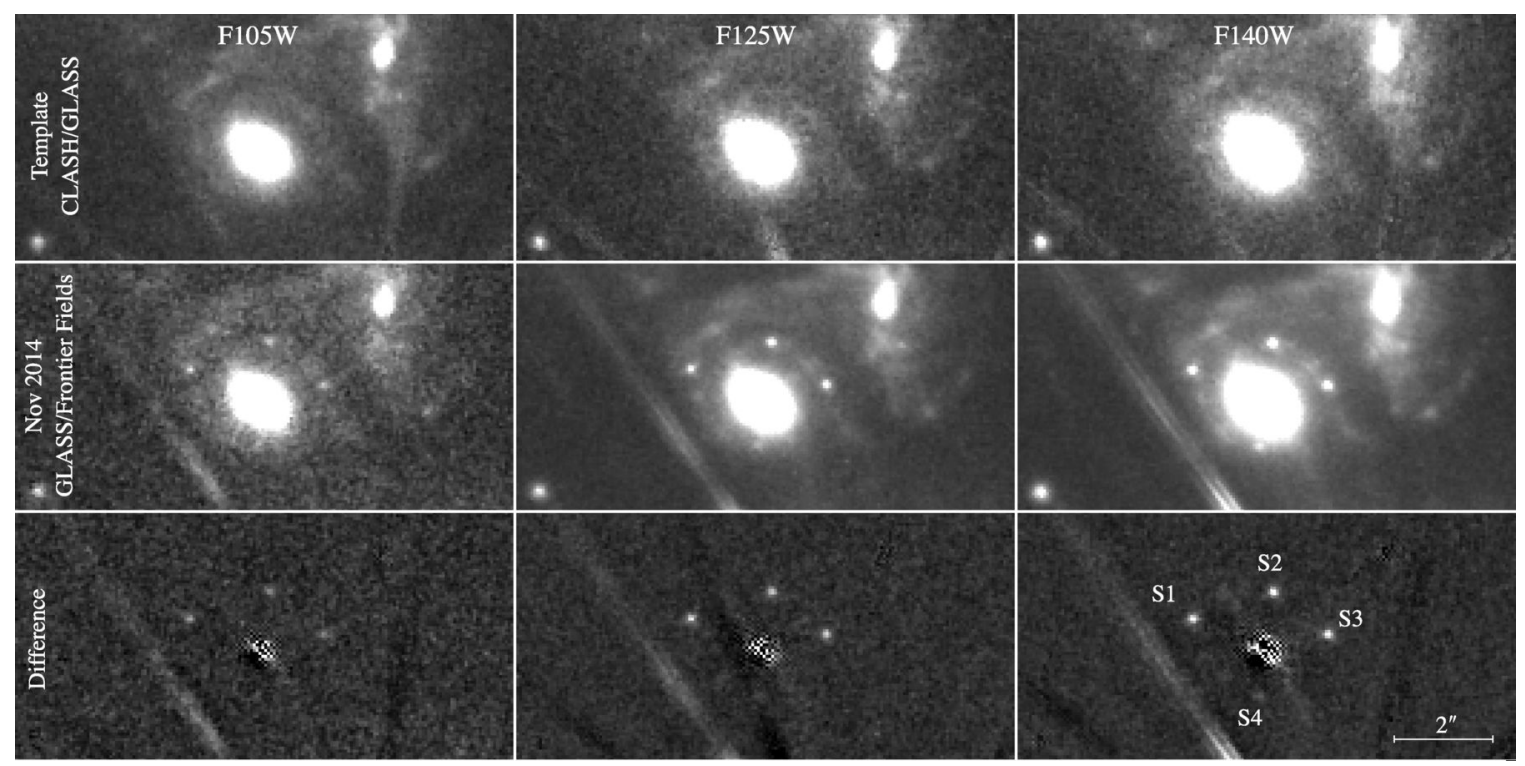

Figure 2: Frames obtained with HST WFC3 of a supernova gravitationally lensed into multiple images by an early type galaxy cluster (Figure from Kelly et al, 2015, [2]).
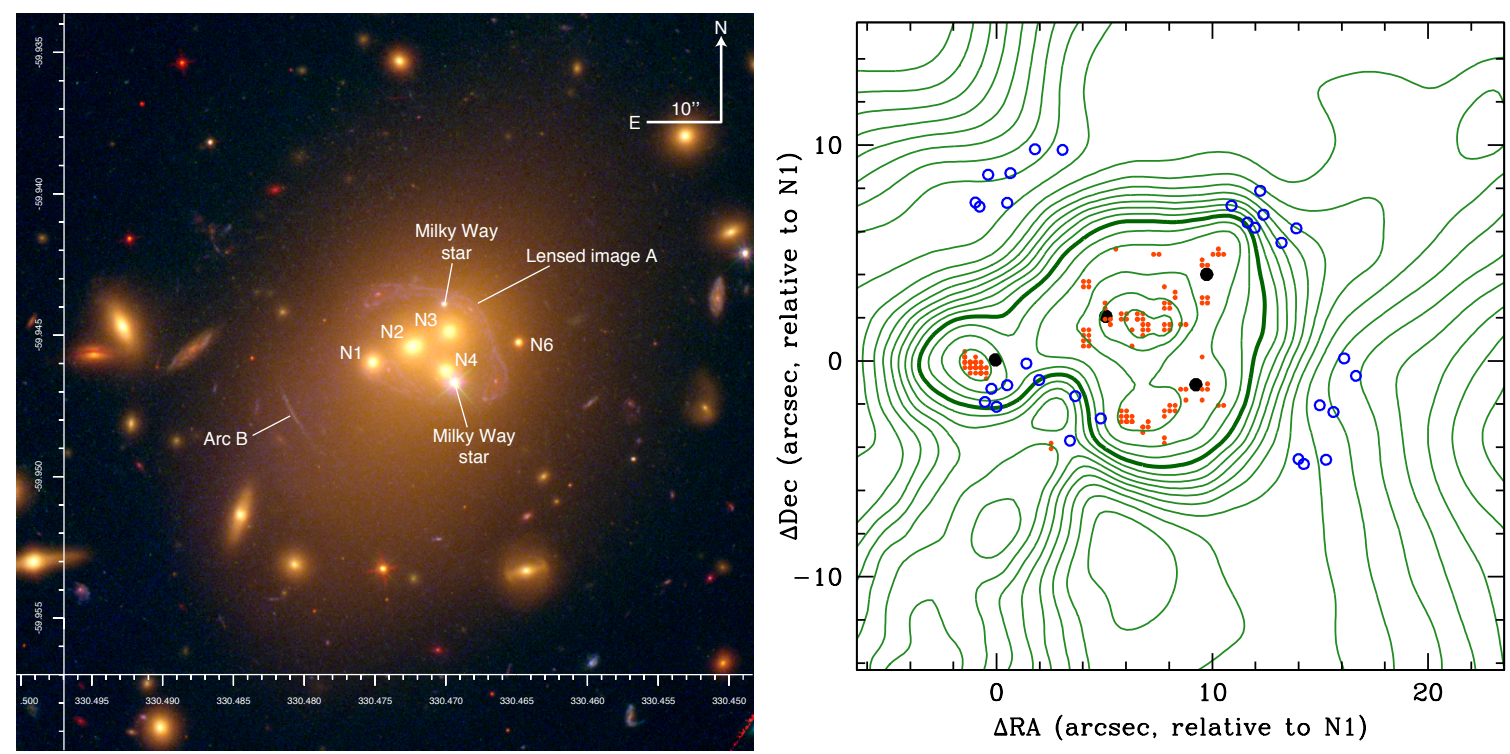

Figure 3: Left: The galaxy cluster Abell 3827, showing gravitationally lensed arcs of background galaxies. Right: The mass density of the cluster derived using lensed image positions (Figures from Massey et al., 2015, [3]). 
full inventory that includes very faint cluster members has been a limitation so far. Synergistic programs including X-ray observations of the hot gas will help to complete the inventory of baryonic materials.

\subsection{Cosmology from clusters of Galaxies}

Galaxy clusters act as strong gravitational lenses. Many of the strong lensing arcs of background galaxies have widths below $0.1 "$ and therefore adaptive optics (AO) based imaging is required to resolve and separate them from the background sky. The strongly lensed region around typical clusters at redshifts of $\mathrm{z} \sim 0.5$ spans $\geq 30$ ", see Figure 3 . Present telescopes cannot provide the required fidelity of observations, the sensitivity and spatial resolution provided by TMT is needed to measure the redshifts of the arcs and to detect substructure in the arcs due to substructure in the density profile of the lensing cluster.

\subsection{Active Galaxies and Black Hole Growth}

\subsubsection{Seeds for Super Massive Black Holes}

A few bright quasars are observed at redshifts of $z>6$, this tells us that black holes with masses of about $10^{10} \mathrm{M}_{\odot}$ formed early in the history of the universe, within the first billion years. Using an integral field spectrograph unit (IFU) (see Section 3.1.1.1) to obtain high spatial resolution spectroscopy of quasars at $\mathrm{z}>6$ we will be able to differentiate between the light from stellar populations and that from 'super-Eddington' accreting intermediate black holes (IMBH). Measuring the metal abundances, gas kinematics and outflows in the quasar host galaxies will help us to better understand fueling, accretion rates and the formation of massive black holes at early times.

In the local universe we can carry out near-IR IFU observations of all types of nearby galactic centers to investigate how SMBHs are fueled and how they control the evolution of their host galaxies

One source of the initial seed black holes in the early universe could be from massive stars. Spectroscopy of galaxies at redshifts $\mathrm{z}>2$ will let us investigate whether the initial mass function (IMF, i.e. the range of stellar masses) evolves over cosmic time. Importantly, evidence for a topheavy IMF at higher redshift could provide a source of intermediate mass seed black holes that could grow to form quasars.

\subsubsection{Central regions, Dusty Torus and Black Hole Mass}

The SMBH sphere of influence can be resolved in diffraction limited observations with TMT and the bright central AGN point source isolated. Diffraction limited spatially resolved spectroscopy with $R<4000$ in the near-infrared with the TMT of many AGN will have the spatial resolution to allow stellar-dynamical analysis that yields the mass of the central massive black holes, $\mathrm{M}_{\mathrm{bh}}$. By collecting many measurements we can trace the growth history of typical $10^{7}$ to $10^{8}$ solar mass SMBHs at high-redshifts in moderate or low luminosity AGNs.

We will be able to constrain the size of the broad-line region, $R_{B L R}$ and probe the structure of the accretion disk region using the technique of reverberation mapping. This technique uses timedelays of the response of broad emission lines to continuum variations and requires rapid optical 
and mid-IR observations with $\mathrm{t}_{\mathrm{samp}} \sim 10 \mathrm{mins}$. Such short sampling times are beyond the reach of existing telescopes.

The outflows induced by AGN winds and jets that prevent runaway star formation in massive galaxies at $\mathrm{z} \sim 0.5-2$ can be observed using near-IR IFU spectroscopy. Synergistic observations with ALMA of molecular outflows and with the VLA of synchrotron emission from jets and outflows are needed to give a complete picture of AGN feedback at redshifts of $\mathrm{z} \sim 0.5-2$.

AGN may have a 'clumpy' dust torus whose properties are strongly affected by AGN activity and the availability of fuel for the AGN. Mid-IR high spatial resolution diffraction limited observations with the 30m TMT will be able to resolve the dust torus in the center of galaxies at redshifts up to $\mathrm{z} \sim 0.5$ or 5 Gyr. Further synergistic observations with ALMA will probe the outermost (cold) regions of the torus structure. High spatial resolution NIR polarimetric imaging will show the regions where the effects of magnetic fields effect are strongest. Polarimetric observations can be used to minimize the dilution of the polarized AGN flux by host galaxy starlight and allow the study of the evolution and morphology of the material obscuring the AGN core.

\subsubsection{Blazars}

Blazars are a sub-class of Quasars or AGN whose emitted jets happen to point toward Earth. Particles in those jets are accelerated to relativistic energies. The acceleration mechanism may be by shock waves, turbulence, and/or magnetic reconnection, the efficiency of each mechanism depends strongly on magnetic field geometry. Also, each mechanism will show different timevariable linear polarization on time scales down to a few minutes.

To explore the acceleration mechanism we will need simultaneous observations of a Blazar with TMT and the Cherenkhov Telescope Array (CTA). TMT will be used to gather moderate resolution optical and mid-IR spectra of nearby AGN on timescales of a few minutes or less. Simultaneous observations will let us relate the magnetic field direction to the acceleration of the highestenergy electrons. Models of the acceleration mechanisms predict micro-variability on timescales $<30$ s at wavelengths observable with the TMT. A full investigation requires optical and NIR low resolution wide wavelength coverage spectro-photometry with sampling times of $\sim 1 \mathrm{~s}$ to $3 \mathrm{~s}$.

As described Section 2.5.2, the structure of the accretion disk and broad-line region and can be mapped with great accuracy and images of the jet morphology obtained in all wavelengths observable with TMT.

\subsection{Binary Super-Massive Black Holes}

In the hierarchical model of galaxy evolution, a large fraction of elliptical galaxies are expected to have stalled binary black holes as a result of galaxy mergers. Non-active binary BHs in the nearest elliptical galaxies will be brought together due to dynamical friction with nearby stars and their presence should be evident from stellar motions. Once the BHs reach a separation of around $1 \mathrm{pc}$ they are expected to stall due to low stellar densities. TMT with adaptive optics will be able to resolve the expected stalling radii of binary BHs and reveal active dual nuclei, see Figure 4. Dual AGN systems may be buried inside the obscuring dusty structure and NIR (1$2.5 \mu \mathrm{m}$ ) polarimetric imaging with TMT will be needed to reveal the conical centrosymmetric polarization pattern centered on each AGN. 

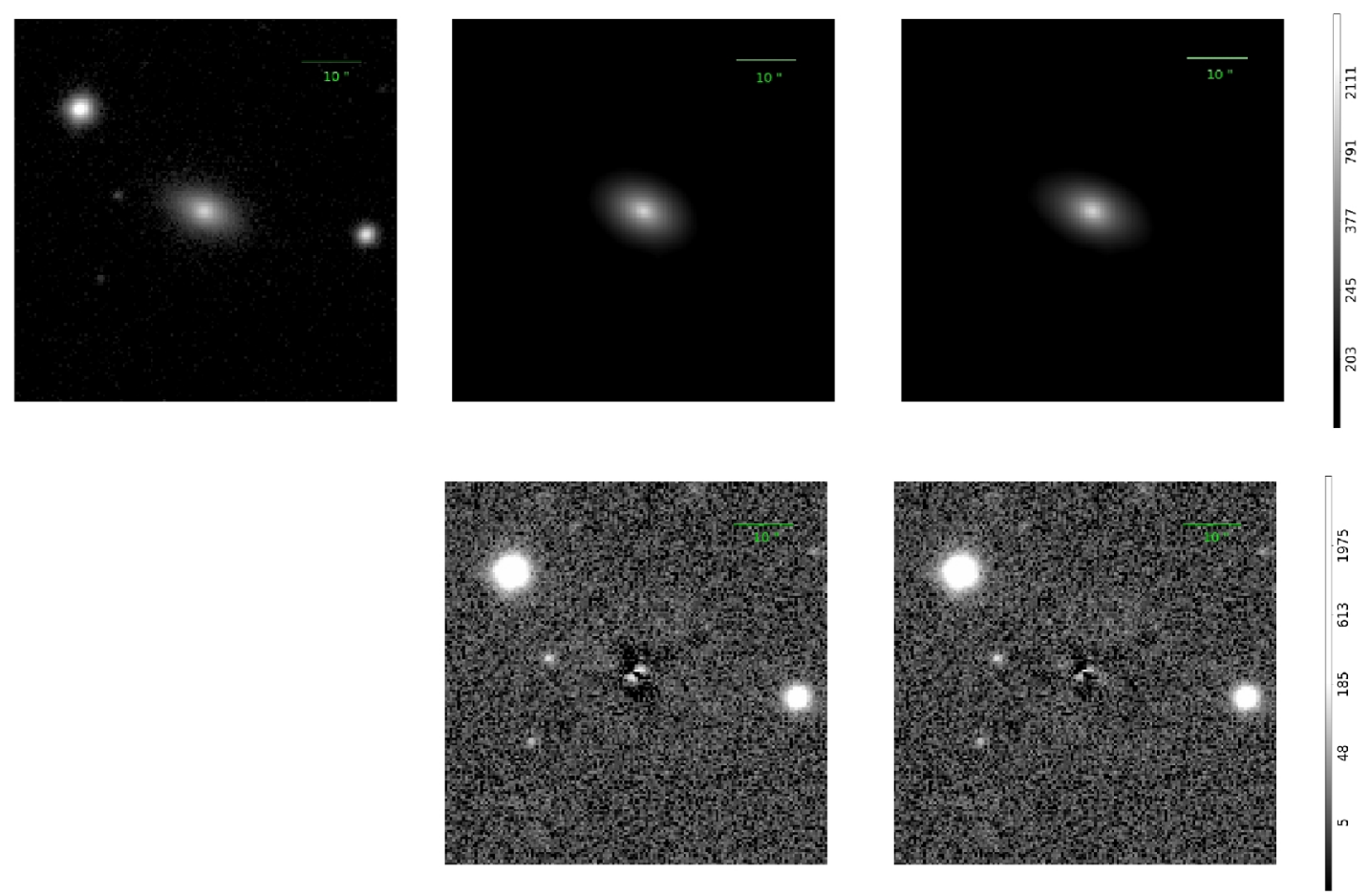

Figure 4: Top left: Original JVLA image of galaxy 2MASXJ1203. Top center right: Model of the galaxy image profile assuming a simple elliptical galaxy profile (center) and with the addition of an exponential term (right). Lower center and right: Residual after subtracting of the model profiles reveals two cores (Figure from Rubinur et al., 2017, [4]).

\subsection{Tidal Disruption Events}

A Tidal Disruption Event (TDE) occurs when a star makes a close approach to an SMBH and is ripped apart by tidal forces. TDEs provide a means to measure the mass and spin of black holes in optically 'normal' galaxies, i.e. galaxies that do not show AGN activity. The disrupted stellar material forms an accretion disk around the SMBH. Soft X-ray and UV $10^{5} \mathrm{~K}$ blackbody radiation from the inner of the accretion disk is reprocessed from the inner accretion region into optical radiation. Many TDEs will be found in large-area surveys such as the Large Synoptic Survey Telecope (LSST) and followup observations will require Target of Opportunity (ToO) response times with TMT of $<1 \mathrm{hr}$ for optical spectroscopy $(\mathrm{R} \sim 2000)$ and 1-2.4 $\mu \mathrm{m}$ spectroscopy $(\mathrm{R} \sim 500)$ that is simultaneous with CTA.

TDEs will be visible by TMT to $\mathrm{z}>6$, less than $1 \mathrm{Gyr}$ after the Big Bang, enabling constraints on SMBH properties and evolution over a vast range of cosmic time. TDEs in nearby galaxies with independent measurements of $\mathrm{M}_{\mathrm{bh}}$ (such as from the $\mathrm{M}-\sigma$ relation or maser disk kinematics) will provide a better calibration of the models for TDEs.

\subsection{Gamma Ray Bursts}

\subsubsection{The Explosion}

The suggested fire ball model for Gamma Ray Bursts (GRBs) is an inhomgenous relativistic outflow with inverse Compton or synchrotron emission, internal velocity variations that cause $\gamma$ - 
ray emission and mild internal shocks at larger radii that cause optical emission. Flux variations of a factor 10 over 1 minute need to be sampled. Consequently, studies of the GRB fireball require prompt observations, beginning within a few hundred seconds of the trigger detection from space based facilities. The combined telescope and instrument response time needs to be $<10$ minutes.

To track the rapid changes in the flux from the fireball, the light gathering power of TMT is needed to provide low resolution spectroscopy ( $\mathrm{R} \sim 100)$ in the optical to NIR (400nm to $2.2 \mu \mathrm{m}$ ) with the required rapid $1 \mathrm{~s}$ sampling. To investigate asymmetries in the shape of the fireball we will need 100s exposure time high signal to noise spectro-polarimetric measurements.

\subsubsection{Progenitors}

There are two forms of GRBs that are observed and different ideas for the cause of GRBs of each form. The rarer short GRBs, that last $<2$ s, are thought to be the result of neutron star mergers. The majority long GRBs, that last between 2 to 1000 s, are thought to be collapsing massive stars (an important tracer of massive star formation at high $\mathrm{z}$ ).

The idea of collapsing massive stars forming long GRBs has some observational support because some, but not all, long GRBs at low redshifts, $\mathrm{z}<0.5$, exhibit super nova behavior. For redshifts of $\mathrm{z}>0.5$ to 1 , any super nova component will be so faint that they can only be identified and followed with a telescope as large as TMT feeding a NIR AO fed IFU. Followup would need to start within $\mathrm{f} \sim 1$ day and continue for up to 30 days after the GRB.

Optical spectroscopy with TMT of low redshift GRBs will identify any faint associated super novae. At this time, all confirmed long GRB host galaxies have been found to be actively forming stars. The host galaxies also have a low total stellar mass and are bluer than present-day spiral galaxies, implying an age for the progenitors of long GRBs of $<0.2$ Gyr.

Observations of the optical counterparts for short GRBs limit any accompanying supernovalike event to being $>50$ times fainter than normal SNe Ia or hypernovae Ic. However they may be consistent with an r-process powered kilonova. To improve the observational constraints on the form of short GRB progenitors, TMT with a NIR AO fed IFU will be the ideal instrument to perform ToO observations in $\leq 1$ day of any kilonova event associated with short GRBs.

\subsubsection{Probes of High Redshift Inter-Galactic Medium and Inter-Stellar Medium}

GRBs can serve as a background source whose light is affected by absorption as it passes through cool and/or neutral hydrogen gas clouds along the line of sight but located in the dense central regions of the host galaxies, in the vicinity of the GRB. This yields critical information about the chemical enrichment history of galaxies directly in the region where the enrichment occurs. This contrasts with the use of quasars as background targets whose lines-of-sight probe low density regions where materials have been affected by gas injection, infall and mixing.

GRBs can be used to trace early galaxy and star formation that re-ionized the universe as GRBs can be detected with TMT at greater distances than the farthest quasars and early galaxies. Presently, progress at $\mathrm{z} \gg 6$ is limited by a lack of sufficiently bright GRBs but with TMT's large aperture and fast response time for ToO observations we will be able to obtain high signal to noise near-IR spectra of faint GRBs out to very high redshifts, $\mathrm{z}>7$, where the relevant spectral features lie at near-IR wavelengths. 


\subsection{Pre-Main-Sequence and Main-Sequence Stars}

The spatial resolution and sensitivity of the TMT will enable studies of individually resolved stars in star forming regions throughout the local group of galaxies and beyond. This will let us study the stellar populations and determine the initial stellar mass function (IMF) over the entire range of masses, from $\sim 100 \mathrm{M}_{\odot}$ to brown dwarfs of $\ll 1 \mathrm{M}_{\odot}$ through the varied galaxy types in the local group. Present studies are restricted to within the Milky Way due to the limited spatial resolution of exiting facilities, see Figure 5.
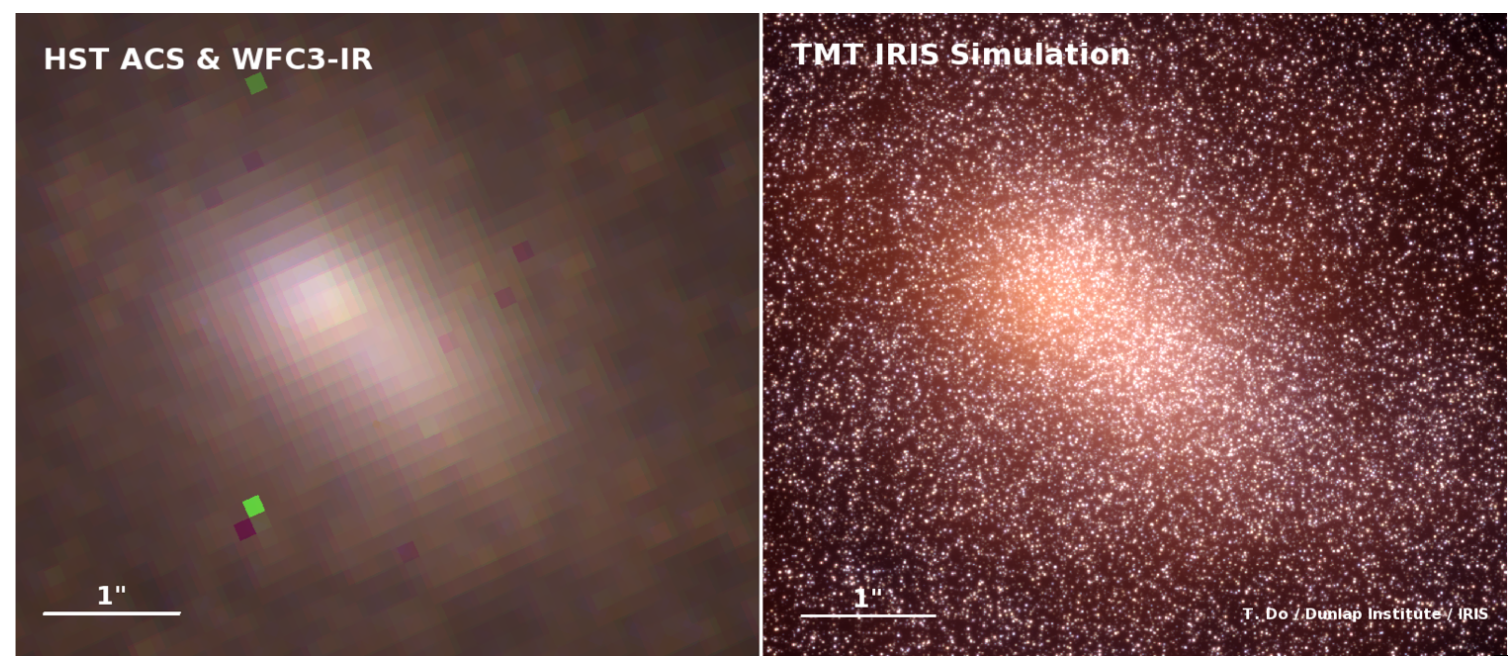

Figure 5: Left: A three color image of the current capabilities using HST ACS (F814W) and WFC3 (F110W, F160W), the limit of the spatial resolution that can be achieved at this time. Right: A simulated image based on our current knowledge of this region, as observed with the Z, J, and K band using TMT's first light instrument IRIS with the NFIRAOS AO. (Credit: Tuan Do, UCLA)

Stellar population studies with TMT will use a variety of AO fed diffraction limited near- and mid-IR IFUs, high resolution spectrometers and high contrast exAO systems. ALMA will provide complementary observations with similar spatial resolution, penetrating the dust to probe the core of dust-enshrouded star-forming regions.

\subsection{Cataclysmic Variables and Novae}

Matter transfer from the Roche lobe filling secondary star toward the heavier compact primary (white dwarf) in Cataclysmic Variables (CVs) and similar types of systems (some have neutron star or Black Hole primaries, some have white dwarf secondaries) often forms an accretion disk around the compact primary. The emission from $\mathrm{CV}$ accretion disks can be mapped and characterized, of particular interest is the variable flickering component that provides important clues about the mechanism for converting gravitational energy to radiation and the mechanism for angular moment transport out from the disk. This mechanism for angular momentum transfer is poorly understood yet it is perhaps the most important astrophysical process, without which no compact structures would form. To map the flickering in $\mathrm{CV}$ accretion disks requires optical spectrophotometric observations $(\mathrm{R} \sim 4000)$ with a high sampling rate $\left(\mathrm{T}_{\text {samp }} \sim 50 \mathrm{~ms}\right)$ of systems that exhibit eclipses. With the TMT, about 100 eclipsing systems can be studied with high signal to noise, representing 
a range of mass transfer rates and accretion disk behaviors. Such observations cannot be obtained with existing telescopes.

Novae are CV type systems that undergo periodic nuclear burning of the material accreted by the white dwarf primary. Novae timescales range from years (recurrent novae) to millennia (classical novae). Novae have polar-blob, equatorial-ring geometry and/or multiple blob like condensations in their ejecta shells. The non-spherical Nova ejecta shell geometry means that the explosive process on the compact star surface is not symmetric. Important aspects of the ejecta activity occur at the time of outburst and the early forming fireball shell provides important information on the burning process on the white dwarf surface. Because of the excellent spatial resolution of TMT with diffraction limited adaptive optics, outbursting novae within a few kpc will become extended objects one week after outburst, the physics occurring within the fireball can be probed with a nearIR IFU. TMT will need to carry out Target of Opportunity (ToO) observing programs that gather optical to mid-IR spectrophotometry and spectropolarimetry $(\mathrm{R} \sim 5000-30,000)$ to characterize the asymmetry and follow the fireballs evolution.

\subsection{Supernovae}

\subsubsection{Characterizing high-z SNe Ia: Towards a Better Standard Candle}

Observations suggest that high-velocity (HV) SNe Ia (V(SiII) $>12,000 \mathrm{~km} / \mathrm{s}$ ) likely originate in the inner regions of higher-luminosity galaxies from metal-rich progenitors that have undergone substantial chemical evolution whereas normal-velocity (NV) SNe Ia (V(SiII) $\sim 10,000 \mathrm{~km} / \mathrm{s})$ appear to originate in less luminous lower metallicity galaxies. Metal-enriched environments decrease with increasing redshift due to the creation of heavier elements being dependent on stellar processing. There may be an evolution of the ratio of HV to NV SNe Ia with redshift.

Testing for redshift evolution of SNe Ia requires the TMT because better spectroscopic classification at high redshift is needed than can presently be obtained. $10 \mathrm{~m}$ class facilities can only obtain high-quality spectra for SNe Ia below $\mathrm{z}=1.5$ however AO assisted near-IR spectroscopy on TMT will easily obtain spectra with better wavelength coverage of SNe up to $z=4$. Diffraction limited AO fed near-IR IFU observations will spatially resolve the components of the host galaxy and provide information such as the star formation rate (SFR) in the host galaxy.

\subsubsection{Unveiling The Explosion Mechanism of Type Ia Supernovae}

To carry out high precision cosmology using SNe Ia requires deciding between the single vs. double degenerate explosion mechanism, i.e. between a white dwarf accreting from main sequence star versus accreting from another white dwarf. Each mechanism creates different amounts of metals in the outermost ejecta that create different spectral signatures. Observations tell us that the outer most layers of the ejecta in NV and HV SNe Ia are chemically different.

To identify the SNe Ia explosion mechanism and test for differences in the early ejecta composition we need to obtain early-phase near-IR spectra of C and $\mathrm{Mg}$ features in a sample of high- $\mathrm{Z}$ SNe Ia. Observations need to begin within 2 days of the beginning of the explosion and have a cadence of a few minutes. Also, late-time optical and near-IR spectroscopy, 1-2 years after the explosion will be a strong diagnostic of the ejecta kinematics and thermonuclear products of the original explosion. 


\subsubsection{Identifying The Shock Breakout of Core-Collapse Supernovae}

In core-collapse $\mathrm{SNe}$, as a result of the collapse of the core, a shock wave propagates outward, emerging from the star as a sudden fireball, the brightest part of a supernova, 'shock breakout'. The shock breakout has a longer duration and redder color for a progenitor star with a larger presupernova radius. Durations range from a few seconds to 1 day and emission peaks in the soft $\mathrm{X}$-ray to UV. Breakouts can be detected at $\mathrm{z}>1$ and can used as a means to probe the high redshift star formation rate because the massive stars that succumb to core collapse have very short lives ( $\leq 10 \mathrm{Myr}$ ). Shock breakouts at $\mathrm{z}>2.5$ will be detectable in $8 \mathrm{~m}$ wide angle surveys such as with LSST and Subaru HSC. The expected decline of a core collapse SNe is $\sim 0.1 \mathrm{mag} / \mathrm{hr}$ at $\mathrm{z}=2$ with a quasi-black body spectrum and so rapid ( $<1$ night) ToO optical spectroscopy $(\mathrm{R} \sim 500)$ of shock breakouts with TMT with 1-2 hr sampling are needed to give the temperature evolution. Slower cadence observations ( 4 to $8 \mathrm{hr}$ ) for the next 2 to 3 days will show the spectral evolution as the metal lines gradually become prominent. This will provide clues about the progenitor such as the pre-supernova radius, CSM structure and mass loss at the last stage of the evolution before the $\mathrm{SNe}$ occurred.

\section{Summary of the Project Timeline and Planned Capabilities}

\subsection{Science drives the design}

The design of entire observatory has been developed to provide the capabilities to carry out a broad range of science. It is recognized that the observatory will be used for science programs that we cannot yet imagine and the telescope will need to host future instruments that we have not yet conceived. The TMT Detailed Science Case [1], that was compiled from the input of the TMT International Science Development Teams (ISDTs), is broad and ambitious and forces the design of the observatory to be flexible and able to support many observing modes. Because of the high quality optics, agility and large instrument platforms of the telescope itself, the types of future instruments that can be supported is relatively unconstrained.

A capability that is of particular interest to astronomers interested in many high energy sources and processes is the requirement for the TMT observatory to be able to begin taking science data within 10 minutes using any instrument in any part of the sky. This rapid response imposes challenging requirements on the telescope structure (for slewing), the tertiary mirror (for changing to a different instrument), the adaptive optics system and laser guide star (LGS) system (to initialize, optimize LGS positions and close the correction loops) and the instruments (to initialize and set to the necessary configuration). The operational procedures to support rapid $\mathrm{ToO}$ programs will be considered as the details of the TMT operations plan are developed. TMT has the most stringent rapid response requirements of the three current ELT projects.

\subsubsection{First Light Instruments}

Three versatile instruments were chosen as the first light instruments in addition to a facility near-IR adaptive optics (AO) system, (NFIRAOS) see Table 1. Two of the instruments (IRIS and WFOS) and the AO system are actively being developed, the third instrument, IRMS, is a close copy of an existing instrument and will be developed closer to the time of first light. IRIS and IRMS will be fed by the AO system, WFOS is a seeing limited optical instrument. 


\begin{tabular}{|c|c|c|c|}
\hline Instrument & $\lambda(\mu \mathrm{m})$ & Field of view/Slit length & Spectral resolution \\
\hline $\begin{array}{l}\text { InfraRed Imager and } \\
\text { Spectrometer - IRIS }\end{array}$ & $0.8-2.5$ & $\begin{array}{l}0.5 \text { - } 2.25 " \text { IFU } \\
33 " \text { imaging }\end{array}$ & $\begin{array}{l}\text { 4000-8000 IFU } \\
5-100 \text { imaging }\end{array}$ \\
\hline $\begin{array}{l}\text { Wide-field Optical imager } \\
\text { and spectrometer - WFOS }\end{array}$ & $0.31-1.0$ & $\begin{array}{l}>40 \text { sq arc } \min (>100) \\
\text { Total slit length }>500 "\end{array}$ & $\begin{array}{c}1000-5000(>7500) \\
@ 0.75 " \text { slit }\end{array}$ \\
\hline $\begin{array}{c}\text { InfraRed Multislit } \\
\text { Spectrometer - IRMS }\end{array}$ & $0.95-2.45$ & $\begin{array}{l}\text { 2' field, up to } 120 " \text { total slit } \\
\text { length, } 46 \text { deployable slits }\end{array}$ & $\begin{array}{c}4660 \\
@ 0.16^{\prime \prime} \text { slit }\end{array}$ \\
\hline $\begin{array}{c}\text { Deployable, multi-IFU, } \\
\text { near-IR spectrometer - IRMOS }\end{array}$ & $0.8-2.5$ & $\begin{array}{c}\sim 20 \times 3 " \text { IFUs } \\
\text { over }>5 \text {, diameter field }\end{array}$ & $2000-10000$ \\
\hline $\begin{array}{l}\text { Mid-IR AO-fed Echelle } \\
\text { spectrometer - MIRES }\end{array}$ & $\begin{array}{c}8-18 \\
(4.5-28)\end{array}$ & $\begin{array}{l}3 " \text { slit length } \\
10 " \text { imaging }\end{array}$ & $5000-100000$ \\
\hline $\begin{array}{l}\text { Planet Formation } \\
\text { Instrument - PFI }\end{array}$ & $\begin{array}{l}1-2.5 \\
(1-5)\end{array}$ & $\begin{array}{l}1 " \text { outer working angle } \\
0.05 " \text { inner }\end{array}$ & $\leq 100$ \\
\hline $\begin{array}{l}\text { Near-IR AO-fed echelle } \\
\text { spectrometer - NIRES }\end{array}$ & $1-5$ & 2" slit length & $20000-100000$ \\
\hline $\begin{array}{l}\text { High-Resolution Optical } \\
\text { Spectrometer - HROS }\end{array}$ & $0.31-1.1$ & 5" slit length & 50000 \\
\hline
\end{tabular}

Table 1: First light (above double line) and potential future generation instruments [5]. Goals for capabilities are in parentheses. Types of instruments not listed here may be developed in response to the scientific priorities and needs of the TMT user community.

\subsubsection{Infra-Red Imaging Spectrometer - IRIS}

IRIS, see Figure 6, has two parallel instrument channels, 1) an imaging channel covering 34 "x34" using four 4k by 4k Hawaii 4RG detectors with 0.004 "/pixel, nyquist sampling the $1 \mu \mathrm{m}$ diffraction limit and 2) an integral field spectrograph (IFS) that provides 4 plate scales and 4 corresponding fields of view. The IFS has 2 pixel scales provided using a lenslet array $(0.45$ "x0.51" @ 0.004/pixel and 1.01"x1.15"@0.009"/pixel) and two using an image slicer (1.13"x2.20"@0.025"/pixel and $2.25 " x 4.4 " @ 0.05 " / p i x e l)$.

The IRIS instrument will consist of an imager housed in the upper half of the dewar and the IFS in the lower part. The imager is being developed in Japan, the IFS in California. IRIS is fed from the first-light adaptive optics system the Narrow Field InfraRed Adaptive Optics System (NFIRAOS). The instrument is attached beneath NFIRAOS, see Figure 7.

3.1.1.2 Infrared Multi-object Spectrometer - IRMS The present concept for a near InfraRed Multi-object Spectrograph (IRMS) is based on the design of the existing MOSFIRE instrument at the Keck Observatory and is conceived as a way to provide this capability at low cost and low technical risk on a shorter timescale than developing an instrument with multiple deployable integral field spectrographs and multi-object adaptive optics. The power of the IRMS design comes from the use of a reconfigurable cryogenic slit mask (see Figure 8) and increased sensitivity due to being fed by the NFIRAOS adaptive optics system.

IRMS will provide up to 46 spectra over a 2 arc minute field with a resolution of about 5000 


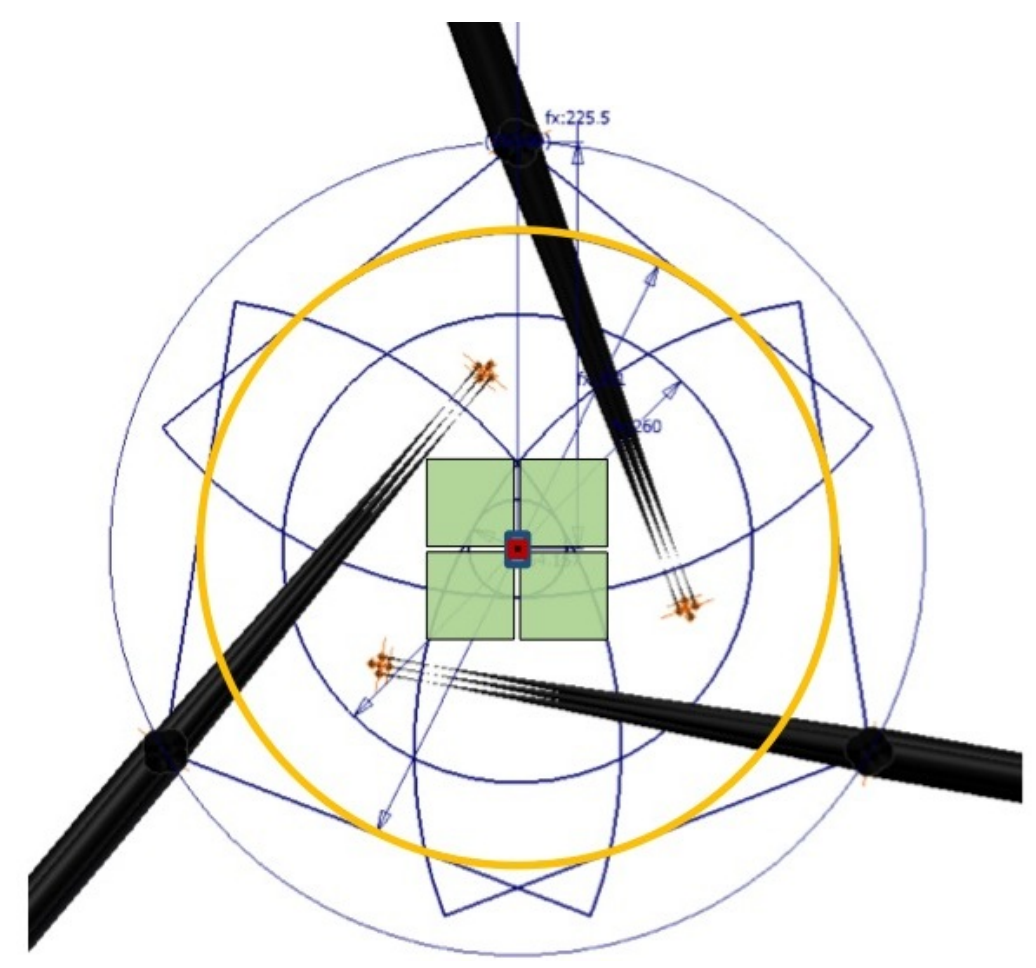

Figure 6: Representation of the focal plane in the IRIS instrument. The four Hawaii 4RG (green squares) create a diffraction limited imager covering 34 "x 34 " with 0.004 " pixels. The fields of view of the IFS are represented by the small colored rectangles in the center. The patrol fields of the on-instrument wavefront sensors (OIWFS) are shown. The yellow circle shows the full field of view of the first light AO system. (Credit: J. Larkin, UCLA)

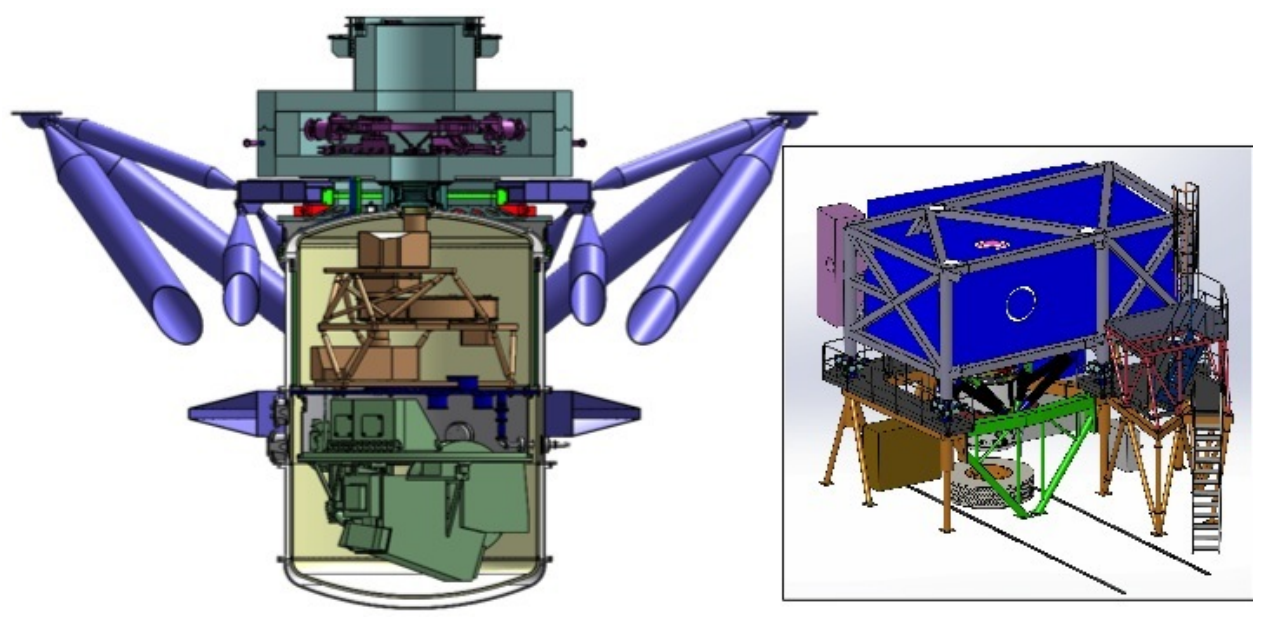

Figure 7: Left: View of the inner parts of IRIS, the IFS in the lower half of the dewar, the imager in the upper half, the 'snout' that contains the OIWFS and the mounting struts to the bottom of NFIRAOS. Right: The large blue box is NFIRAOS and IRIS is seen below with the donut shaped cable wrap on the floor of the instrument platform. (From: J. Larkin, UCLA) 

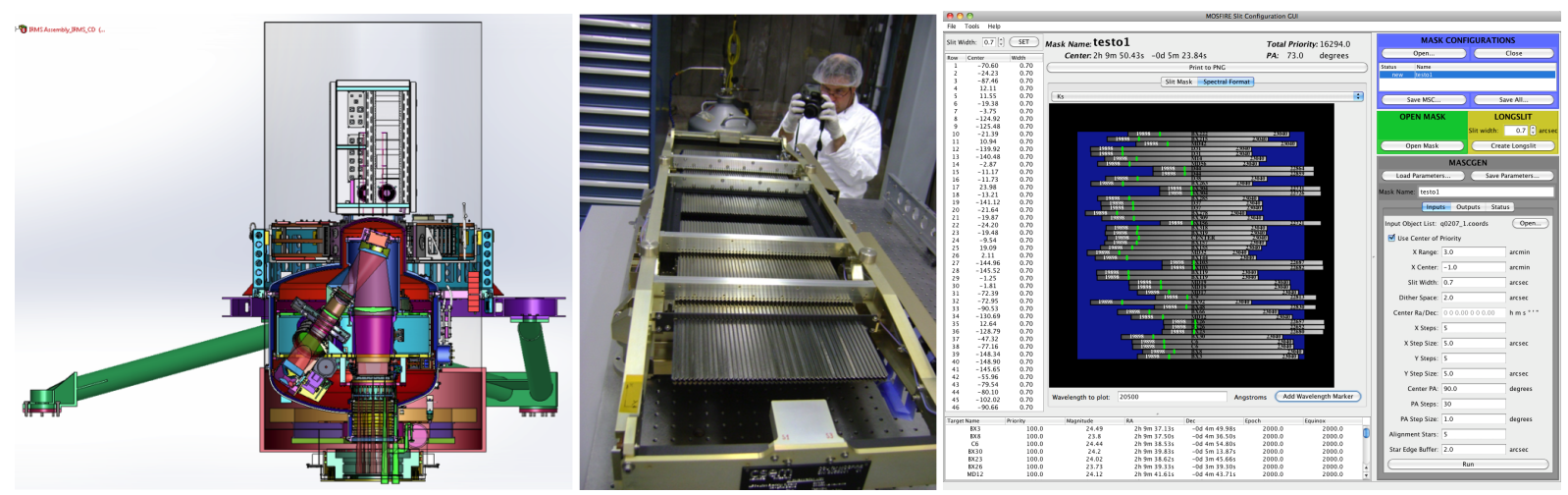

Figure 8: Left: IRMS as installed at the upper port of the NFIRAOS adaptive optics system (From: B. Mobasher, UCR). Center: The reconfigurable cryogenic slit mask used in MOSFIRE at Keck. Right: The slit configuration software in use at Keck that allows masks to be reconfigured in 3 minutes. (From: W.M. Keck Observatory)

covering $0.97 \mu \mathrm{m}$ to $2.45 \mu \mathrm{m}$ with a sensitivity of about 3.3 magnitudes more in $\mathrm{J}$ and $\mathrm{K}$ compared to MOSFIRE.

3.1.1.3 Wide Field Optical Spectrograph - WFOS A multi-object wide field optical spectroscopic capability has a high scientific priority for TMT. Several design concepts are being explored at this time, including a deployable fiber fed system, image slicers and a more traditional system utilizing pre-made slit masks. A baseline design that was previously developed is described here to illustrate the capabilities that can be expected for WFOS regardless of the final design.

The MOBIE (Multi-Object Broadband Imaging Echellette) design for WFOS was developed several years ago (see Figure 9) and provides spectral resolution up to $8000,>40$ sq. arc min field of view, multiplexing capabilities from a single target up to 200 and wavelength coverage from $0.31 \mu \mathrm{m}$ to $1.1 \mu \mathrm{m}$. Programs would trade off the wavelength coverage, number of targets and resolution. The system would also work as an optical imaging camera.

\subsubsection{Development plans for 2 nd generation instruments and existing ideas for instrument types}

In order to ensure that new capabilities arrive at the observatory every two to three years, an instrument development process has been established (see Figure 10). The beginning of the process is based on community explorations that identify the scientific priorities and required instrument capabilities of the TMT user community. Subsequent stages involve initial technical exploration and down selection followed by a standard instrument development process. The process is flexible, there is no predefined instrument delivery sequence, the process can respond to changes in the scientific interests of the TMT user community.

The planned construction timescale for the TMT is approximately 10 years and the development timescale of a facility class instrument for TMT is about the same. Consequently the first call for proposed 2nd generation instrument studies was released in September 2017. 


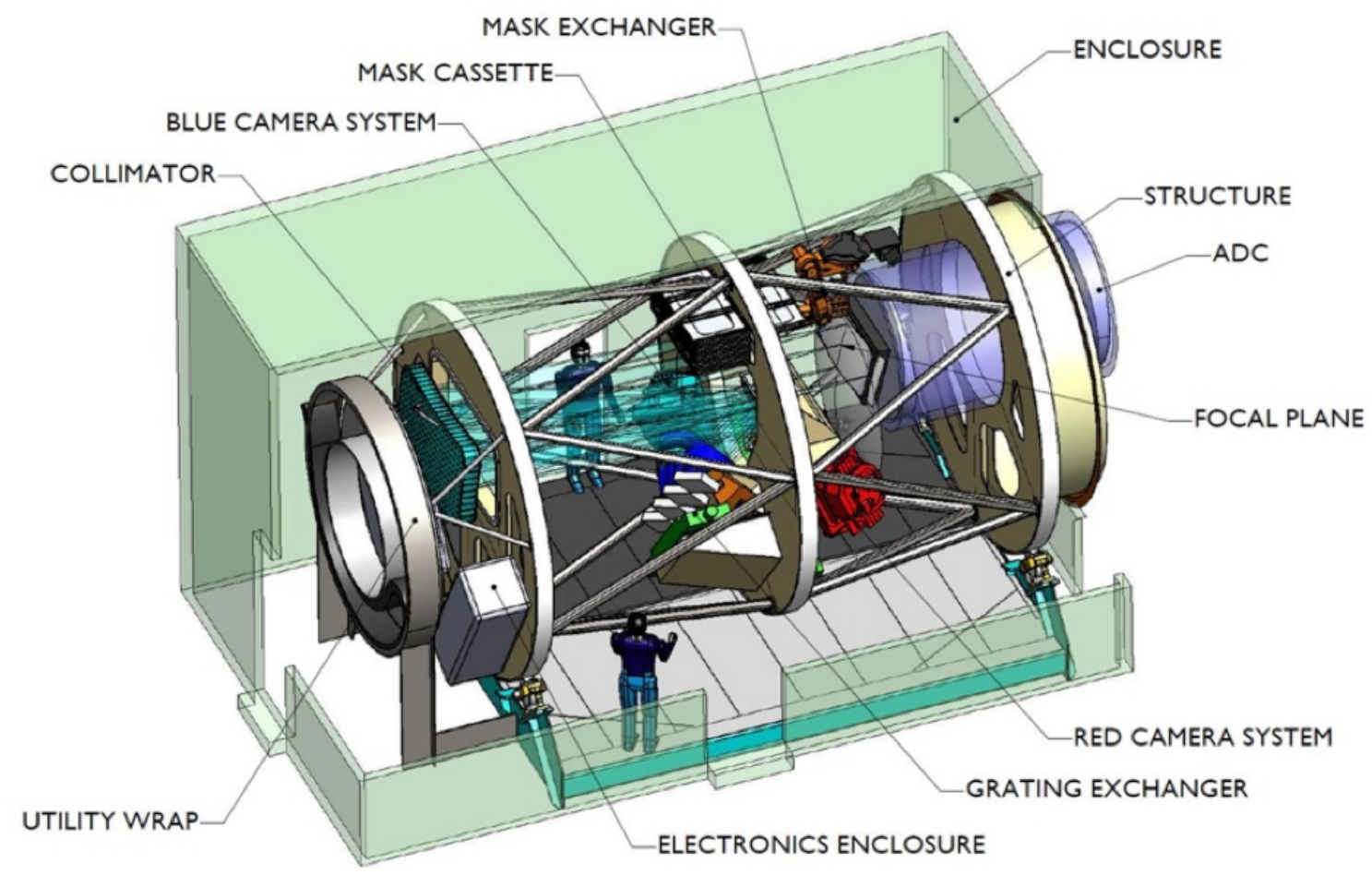

Figure 9: Image of the MOBIE design for WFOS. Light enters from the right. The large scale of the instrument is seen. A significant technical challenge for this design is the fabrication of optics that are large enough. (Credit: TMT International Observatory)

\subsubsection{Queue scheduling and Target of Opportunity Observations}

Of particular relevance to studies in many areas of high energy cosmic sources is the ability for an observatory to respond rapidly and carry out Target of Opportunity (ToO) observing programs.

Rapid response depends on two factors, 1; the physical ability of the observatory to slew, change and configure instruments and for many cases the rapid delivery of adaptive optics corrected images to the instruments, 2; having the appropriate operational procedures in place to pre-define ToO programs and respond rapidly (ideally automatically) to alerts.

As previously mentioned, TMT has the most stringent requirements for rapid response of all of the ELTs with a 10 minute maximum response from ending one observation to beginning science observations on another target at a different position with a different instrument. This is a critical capability for studies of many types of transient objects. The observatory is being designed to support queue scheduled observations and that ability allows the possibility of rapid execution of a ToO program by interrupting the queue.

\subsubsection{TMT Construction Timeline}

Maunakea on Hawaii Island was selected in 2009 as the site for TMT after a long site testing and selection process. TMT began exploring construction in Hawaii in 2003 (whilst investigating several candidate sites) and establishing local employment, workforce development and education 


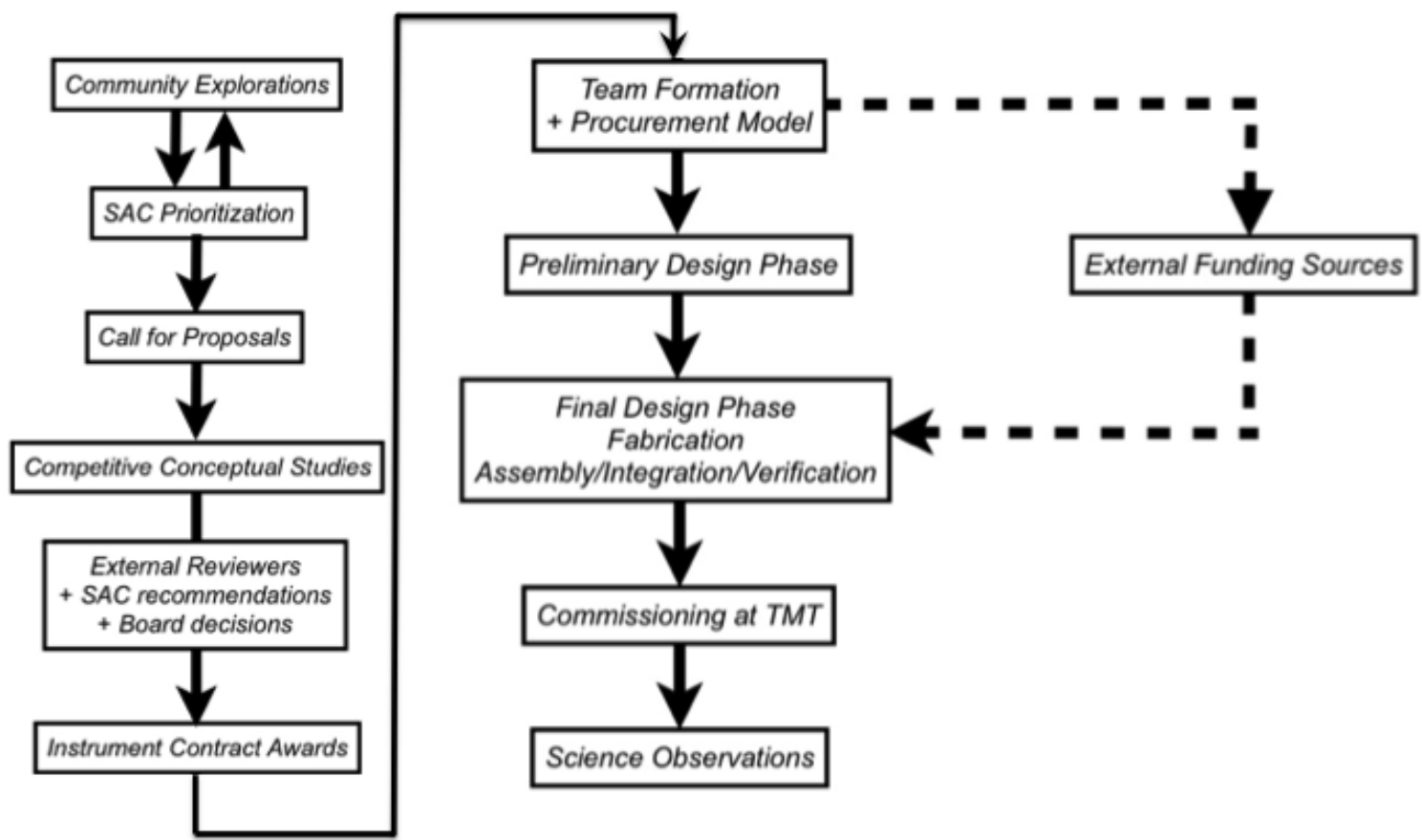

Figure 10: Flowchart showing the instrument development process for post-first light instruments for TMT [6]. This process will be repeated every 2 to 3 years to ensure a regular arrival of new instruments to the observatory. The first cycle is beginning now as the development timescale of about 10 years is the same as the TMT construction timescale.

programs from about 2009. The project entered the construction phase in 2014 but protests and the revocation of the building permit at the end of 2015 have created delays.

In order to move forward, in 2016 a call for proposals to host TMT was distributed and after considering the possible alternate sites a final selection of Observatorio del Roque de los Muchachos (ORM) on the Island of La Palma as the alternate site for TMT was made.

On-site construction has been rescheduled to restart in early 2018 at either Maunakea or ORM. For both sites, the full legal processes of seeking the necessary permissions to begin construction are being followed at this time and the final decision of which site will be made closer to the construction restart date.

Construction is scheduled to last about 10 years and will transfer to operations when the telescope and some of the first light instruments are delivered and science commissioning has begun. This is planned for the late 2020s.

\section{Conclusions}

The Thirty Meter Telescope will provide exciting capabilities that will enable many different studies of high energy astrophysical sources and high energy processes over small to cosmic size scales. Strong synergies will exist with other astronomical facilities, be they survey telescopes or facilities that operate at other wavelengths, gravitational wave observatories, neutrino or cosmic 
ray detectors. The rapid response capabilities and range of instrument capabilities will create a versatile and powerful tool for the exploration of the high energy universe.

\section{References}

[1] Skidmore, W., 2015, "Thirty Meter Telescope Detailed Science Case: 2015", RAA, 15, p1945

[2] Kelly, P., et al., 2015, "Multiple images of a highly magnified supernova formed by an early-type cluster galaxy lens", Science, 347, p1123

[3] Massey, R., et al., 2015, "The behaviour of dark matter associated with four bright cluster galaxies in the $10 \mathrm{kpc}$ core of Abell 3827", MNRAS, 449, p3393

[4] Rubinur, K., Das, M., Kharb, P., Honey, M., 2016, "A candidate dual AGN in a double-peaked emission-line galaxy with precessing radio jets", MNRAS, 465, p4772

[5] Simard, L., et al., 2010, "The TMT Instrumentation Program", SPIE, 7735, 23-1

[6] Simard, L., et al., 2015, "Planning Future TMT Instruments", TMT2G Workshop http://goo.gl/MwzMHV

\section{DISCUSSION}

Paul Mason: Will the instrument platform move with the mirror or will it remain stationary?

Warren Skidmore: The instruments will all be located on the Nasmyth platforms at the sides of the telescope, these platforms are part of the telescope structure and will move in azimuth around the vertical axis of the center of the telescope. This provides a constant vertical gravity vector for the instruments. When the telescope moves in altitude to follow an object, the tertiary mirror (located in the middle of the primary mirror) will track and send the beam to which ever instrument is selected. 